\title{
Accessibility in STEM: \\ Barriers facing disabled individuals in research funding processes
}

\section{Executive Summary}

Disabled researchers are a key underrepresented group within the STEM community. While they make up $4 \%$ of the national UK academic community, no data is currently openly available to assess the representation of disabled researchers and their success rate in STEM disciplines. Recently, UKRI data has been released that shows only $25 \%$ of disabled researchers apply for research grant funding across all disciplines, with the average success rate and award amount consistently lower for disabled researchers compared to non-disabled researchers. To understand this clear imbalance and disparity between disabled and nondisabled researchers, representatives from this underrepresented have been consulted to determine barriers that prevent them from obtaining research funding. This report summarises the direct barriers at each stage within the research funding process, as well as indirect barriers that have an adverse effect on their ability to obtain research funding. It aims to raise awareness of issues facing disabled researchers and provides suggested actions for improving accessibility within the research funding process.

\section{Introduction}

The representation of disabled researchers in STEM remains low - with representation falling by $\sim 40 \%$ from undergraduate to postgraduate level in physics and engineering. At staff level, it is unknown whether this 'leaky pipeline' continues, as no evidence currently exists as to the representation of disabled individuals in STEM at this stage, as it has not been currently captured. However, of the total academic staff within HE institutions only $4 \%$ are disabled, of which $5 \%$ are deaf or hearing-impaired and $3 \%$ are blind or visually-impaired [1]. Increasing the number of disabled individuals studying, working and leading in STEM subjects will have a huge impact on the UK economy.

Out of the UK working-age population in 2018, approximately $19 \%$ of individuals possess a known disability. A $10 \%$ rise in the employment rate amongst disabled adults would contribute an extra $£ 12$ billion to the Exchequer by 2030 [2]. EngineeringUK have reported an annual shortfall of 59,000 graduates needed to fill core engineering roles. They estimate that an extra 1.2 million engineers are required by 2025 to meet the UK's economic needs. It is therefore essential that underrepresented groups, including disabled individuals, have all the support necessary to enter and succeed in the STEM workforce.

Diversity within the workforce has been shown to increase productivity and innovation [3]. It is therefore in the national interest to ensure equality and diversity within the STEM community, if the UK wishes to remain world-leading in research and innovation. Disparity in research funding has a detrimental impact not only on an individual's career progression and stability but also their ability to conduct world-leading research. Disabled researchers face several direct and indirect barriers in applying for or obtaining research funding that place them at a significant disadvantage to other groups.

However, when assessing the representation of disabled researchers within the UK research community, data on application rates, research themes and success rates for this group could not be obtained. While such data has been provided for other underrepresented groups (e.g. women, BAME), data pertaining to disabled researchers has not been extensively collected or analysed. This significant lack of data highlights both the lack of representation and awareness of disabled researchers within the research funding process. By not engaging with this important stakeholder group and also lacking vital information around their success in obtaining research funding, inaccessibility in the process cannot be addressed. 
Recently, the UKRI released some data pertaining to disabled researchers in response to a request from the House of Commons Science and Technology Committee. While limited in its scope, this initial data showed that disabled researchers make up only $1 \%$ of principal investigator applicants across UKRI. Considering national representation of disabled researchers in academia (4\% from HESA 2016/17 [1]), only $25 \%$ of disabled researchers are applying for research funding. This points to significant barriers to applying for research funding at the early stages of the process. Most notably, disabled applications were found to consistently win smaller awards than non-disabled counterpart, with the mean award amount awarded to disabled applications less than half that for non-disabled applicants in 2018/19 [4]. As disabled applicants have higher costs associated with travel, research equipment and research support, this particular statistic is alarming and highlights potential barriers and bias within the research funding process.

Following consultation with members of this underrepresented group, this document aims to summarise barriers and unconscious bias facing disabled researchers within research funding processes. It will address both direct and indirect barriers and provide suggestions for future actions to ensure accessibility in STEM research funding.

\section{Direct barriers within the research funding process}

Disabled researchers face direct barriers to accessing research funding at all stages of the funding process - from the point of entry to receipt and management of a research award. These barriers are summarised below for each stage of the research funding process.

\subsection{Accessing guidance on grant applications and internal selection processes:}

The UK research councils have several schemes available that are either focused on promoting researchers within a certain career stage (e.g. early career fellowships); proof-of concept research (e.g. Innovate); fostering new interdisciplinary collaborations (e.g. GCRF fund); or pursuing a programme of work within an identified research theme that is expected to have global impact (e.g. programme grants, flagships). To reach a diverse range of researchers, advertisement in accessible format to a broad range of users is crucial. Preparing a high-quality grant proposal also requires extensive research into the aims and objective of the grant. To ensure that the proposal falls within the remit of an open scheme and meets all essential criteria, researchers need to access the guidance for that particular scheme. Disabled researchers have identified that the following barriers to accessing information and guidance for grant schemes:

- Calls for research funding schemes are often advertised only on the research council website or via email and are not offered in an alternative format (e.g. PDF). Advertisement via email usually provide hyperlinks to access the same advertisement on the website and also do not provide alternative formats.

- New research calls can be first advertised on social media sites (e.g. Twitter), which are not as accessible for use with screen-readers. Alternative text and subtitles are also not provided for images and videos, making it hard for disabled researchers to access this information and prepare research proposals with sufficient time.

- Research council websites have not been designed to provide basic accessibility. They do not work well with screen-readers and are hard to navigate.

- Advertisements for schemes and news items are often presented in video format and do not come with an alternative format. Videos posted on the website often do not have subtitles or transcripts provided. When transcripts are available, it requires the researcher to contact the research council directly to request a copy.

- Guidance for specific grant schemes are only provided in an online format or via PDFs. While PDFs can be an accessible format for use with screen-readers, they must be updated to provide basic accessibility. The version provided on research council websites is often in the inaccessible format and must be updated by the researcher themselves. Many official bodies, such as gov.uk, and are working to remove PDFs 
from online spaces [5].

- The majority of graphics and figures on research councils do not have alternative text. As infographics are increasingly used to detail current UK research priorities and future directions, disabled researchers who rely on alternative text when reading figures are losing vital information.

- When searching for information about accessibility on research council websites, it is explicitly stated that PDFs can be altered by the researcher themselves and that the researcher should contact the council directly for help. For example, to access alternative pdfs with basic accessibility or to receive alternative text for figures, the researcher has to contact the council directly. This places the onus of accessibility on the researcher not the council, increasing their workload and barriers to access vital information around the funding process.

- For specific questions on grant schemes, researchers have to contact the research council directly. Often only one means of communication is provided: either phone or email. When only phone contact details are provided, this shuts off a line of communication for deaf/hearing-impaired and/or neurodiverse researchers. When email details are provided, visually-impaired researchers lose out. As disabled researchers frequently need to contact councils directly to receive accessible formats and discuss accessibility issues in the process, a variety of communications are required.

- For some grant schemes and fellowships, research councils enforce universities to undergo a formal selection process to limit the numbers of applicants. Universities therefore undergo a variety of informal internal selection processes to conform with university strategy. As disabled researchers often have additional needs that are not funded or supplied by research councils, extra cost devolves to the university and can lead to disabled researchers not being selected in internal review processes due to this additional cost. Furthermore, internal selection processes typically do not undergo any diversity monitoring and selection panels do not have any disability awareness training. This can lead to an increase of bias within the selection process that can negatively impact disabled researchers.

\subsection{The grant application process:}

While the grant application process can differ between research councils (e.g. page limit, format, etc.), there are key fundamental elements required for all schemes and research councils. For example, each grant proposal requires a description of the proposed research and methodology (case for support) and a justification of resources. The quality of this research proposal determines if the proposed research is deemed suitable for funding or not. The grant application is therefore the most important part of the research funding process, having a significant impact on a researcher's success. The following barriers in accessing the grant application platform and associated guidance therefore drastically reduce the chance of success for disabled researchers and need to be addressed.

- It is important to note that all of the barriers described in $\$ 2.1$ also apply to the grant application process.

- Funding application platforms are often inaccessible. Research councils are increasingly only allowing applications via online portals, which have limited accessibility. For example, the current means for submitting grant proposal to UKRI is via the online je-S system. This system currently does not have any disability tools (e.g. blue/black contrasts, alternative text, ruler on screen) to ensure basic accessibility. The system itself is not compatible with the majority of screen readers and does not have audio descriptions for figures or important announcements.

- The help tools and information for the je-S online system is also only available online and not in accessible format. The online wiki does not have any disability tools to provide basic accessibility and cannot be downloaded into an accessible PDF format. Disabled researchers therefore need to contact the research council directly for help 
or obtain extra assistance to complete the research application.

- Formatting is not consistent across UK research councils, preventing the use of one template for all grant proposals. As disabled researchers often have to use speech-totext software or assistants to compose documents, the ability to use one template for all proposals would drastically reduce the increased time and effort needed to draft a grant proposal.

- Across all research councils, the turnaround time between the announcement of a new grant scheme and the deadline for grant submission is extremely short (on average 1 month). In particular, for a large number of research councils, a response to reviewers is expected within 5 working days of receipt of peer reviews of the grant proposal. While this quick response time is demanding for all researchers, it significantly impacts disabled researchers more, who have other needs and requirements. For example, a researcher could need to arrange time with an assistant to type the grant proposal or assist with using the online platform; or could be on medical leave due to a chronic illness. Short deadlines are inaccessible to individuals with these requirements, impacting on their ability to submit a successful grant proposal.

- UKRI and the je-S system do not have published accessibility statements, which must be published by $23^{\text {rd }}$ September 2020 [5].

\subsection{Peer-review process:}

Following submission, the grant proposal goes through a peer-review process. While the peerreview process aims to be anonymous and assess the quality of the proposed scientific research and methodology alone, the following issues arise:

- The peer-review process is not a blind review process. Personal details, such as gender and age, are not actively hidden, increasing the risk of unconscious bias in the process. The name of the applicant is included in letters of support, description of trackrecord and the host letter of support, so there is no anonymity of the proposer.

- Peer-review of the grant proposal does not just look at the case for support but the whole proposal. In particular, the reviewer is asked to review the justification of resources and comment on the 'value for money' of the proposed research. Disabled researchers often have increased costs for travel and specialised equipment, leading to a much-higher overall cost for the proposed research. Assessment of the 'value for money' of the proposal therefore disadvantages disabled researchers unfairly.

- Peer-reviewers receive no disability awareness training before reviewing a proposal, increasing the risk of unconscious bias affecting the review process. One common misconception is that a disability will prevent a researcher from completing the proposed research or achieving as much success as a non-disabled researcher. This is simply not the case. The only difference is that reasonable adjustments must be provided for the disabled researcher.

- Peer-reviews are not thoroughly sifted or checked for cases of unconscious bias. Comments not related to the proposed research (e.g. personal comments about the proposer) are not redacted from the review and are often passed on to the proposer, which can be upsetting and affect the score given for the proposal.

- Following receipt of peer-reviews, researchers are given a short turnaround time (1 week) to reply to reviews. This short deadline is detrimental for disabled researchers, who need more time to process and respond to the reviews. For example, some disabled researchers require a support worker or use of specialist equipment (e.g. speech to text software) that must be arranged (with $>1$ week waiting time) and can be more time-consuming.

\subsection{Interview process:}

Another key step in the research funding process is the interview. An interview is usually requested for individual fellowships and large-scale research grants (e.g. strategic equipment grant, programme grant) and takes place after peer review to form a second shortlisting stage. 
The interview allows the research council to directly address questions and points raised during the peer-review process to the researcher, providing them a chance to present why their research should be funded. A good performance at interview can ensure the success of a proposal, whereas a poor performance could lead to the proposal not being funded. Consultation with disabled academics has highlighted the following barriers at interview stage:

- Firstly, the interview process is inherently biased. Unconscious bias rewards a certain type of researcher (usually white, straight cis-male and non-disabled) and rates the research proposal on the researcher themselves rather than the quality of the proposed research.

- The interview process does not automatically provide basic provision for disabled researchers (e.g. telecoil loops, wheelchair access). To request reasonable adjustments for interview, researchers must contact the research council directly, where it is stated they will 'assist as much as possible'. Disabled researchers are often expected to arrange the additional support themselves via their employer or AtW scheme. This does not promote an inclusive environment and places an additional burden on the researcher when preparing for interview.

- Research councils does not cover costs for additional care required if the interview takes place in a normal working day or as part of normal care plan. They look to the employer or the AtW scheme to cover costs first and will only reimburse if they find that it is not part of the researcher's normal routine. This makes peer-review panel and interviews challenging.

- Similarly, research councils do not cover the extra cost for travel to interviews. They will not reimburse any extra costs associated with travel and expect the employer or AtW to cover all costs.

- While remote access to interviews has recently been introduced by UK research councils, it is explicitly stated within interview guidance that if the technology does not work within a set time limit ( 5 minutes), the interview will be abandoned. This does not promote an inclusive environment or ensure accessibility for disabled researchers, who often cannot attend in person and rely on remote access to interviews.

- Interviews have a set time limit and are often run to a strict time schedule. Disabled researchers often need more time to prepare and respond to interview questions (e.g. lip-reading takes extra time to process question). However, this extra time is not usually factored in when arranging interviews.

- No breaks are allowed during the interview process for academics with chronic fatigue, as interviews follow a set time limit.

- The representation of disabled researchers on interview panels is minimal and data on panel representation is not recorded or monitored for this protected characteristic.

- Panellists and reviewers often have not received training on unconscious bias and accessibility, so are not aware of the best practise for ensuring basic accessibility. They are also often not informed of the legal requirements (Equality Act 2010) for ensuring that all possible reasonable adjustments are made for disabled researchers.

\subsection{During the grant:}

Following success of a grant application, there are significant steps post-award that must be implemented to receive the research funding and ensure the success of the proposed research. For example, official acceptance of the award is required and researchers must ensure that they are using the funds correctly and recording research undertaken. Barriers are also present at this stage of the process:

- When queries arise during the grant term, researchers have to contact the research council directly to ask for information. Generally, only one means of communication is offered (either phone or email), which poses challenges for disabled researchers (as described in §2.1). 
- Terms and conditions on the use of research grant funding are not provided in an accessible format. They are usually presented in an online format or via PDFs without basic accessibility.

- There is a lack of funding for reasonable adjustments and extra resources required by disabled researchers during the grant, which drastically impacts their ability to achieve high-quality research outputs. In particular, PhD students funded on grants do not receive financial support during their writing-up period for reasonable adjustments. Barriers due to lack of funding are further described in $§ 3.1$ and §3.5.

\section{Indirect barriers impacting research funding}

Alongside the direct barriers to obtaining research funding described in $\S 2$, disabled researchers face several indirect barriers that further impound on their ability to access the research funding process and excel within the STEM community [6-18]. These barriers are often institutional and have a significant impact on the researcher's career progression, track record and ability to produce world-leading research, which in turn affects their ability to attract research funding. These barriers are summarised below:

\subsection{Funding/Resources available on the grant:}

One major challenge facing disabled researchers is the additional cost associated with their required adjustments when performing research. The majority of research councils state that all costs associated with these adjustments should be covered by the employer or the AtW scheme and additional costs associated with implementing the research proposal (e.g. conference travel) will not be reimbursed. While AtW and employers do cover costs for a normal working day routine, research costs often fall outside of this remit. This places the burden of funding adjustments to the disabled researcher, preventing them from taking an active role in research. The restrictions placed by research councils on what costs can be requested and associated barriers are summarised below:

- UK research councils do not cover costs for additional care, for example when attending interviews or conferences. They look to employer to cover these costs first and only reimburse if they find that it is not part of a researcher's normal routine.

- Similarly, extra costs associated with travel to conferences, meetings and field trips are not covered and are expected to be funded by the employer.

- Research fellowships often do not count as formal employment, so disabled research fellows cannot access government-funded schemes, such as AtW to cover the costs of reasonable adjustments.

- If requesting extra funds for care or additional support, disabled researchers have to justify the cost by disclosing their specific disability and requirements at the beginning of the grant process (stage §2.2). This takes away anonymity and does not promote inclusivity. It can lead to embarrassment for the disabled researcher, who must explicitly describe their disability and prove to the council why the costs are justified. The lack of anonymity at such an early stage of the process also leads to unconscious bias, where panellists and reviewers can unconsciously make a judgement on the researcher's physical and mental capability to conduct the research.

- Increased costs for specialised research equipment and software (e.g. to work in conjunction with accessibility software) must be justified by disclosing the specific disability and are often queried or denied by needs assessors. The increased cost of the research grant can also reduce chances of success if the total research funding budget is reduced for that funding round.

- Funding for doctoral candidates often includes a writing-up year without a living stipend, which disadvantages those with higher direct living costs and lower overall earnings arising from reduced earning capacity due to disabilities.

- Funding for doctoral candidates on research grants either does not include costs for reasonable adjustments at all or does not cover costs during the writing-up period. While the Disabled Students Allowance (DSA) can cover some of these costs during 
the $\mathrm{PhD}$, it does not include the writing-up period, leaving several disabled $\mathrm{PhD}$ students unable to complete their doctoral studies. It also does not consider the need to review and maintain equipment once supplied to ensure it carries on being a functional adjustment, preventing disabled doctoral students from conducting the proposed research on the grant.

- While for some research councils PhD funding applicants have the option to apply for a support worker, costs associated with extra supervision time are not allowed. This is detrimental, as universities and supervisors cannot take on disabled PhD students if costs are not recovered.

- At the time of writing, there is no guidance from research councils on applying for costs associated with disability. This deters disabled researchers from applying for grant funding, as their costs cannot be recovered.

\subsection{Medical leave / healthcare}

Disabled researchers often have to take time away from research for medical leave associated with their disability. This can have a substantial impact on their ability to carry out the research, require either extra assistance, use of support workers, increased project durations and/or flexible working schedules. However, the following challenges arise when taking medical leave:

- Medical leave is often not considered as a career break or taken into account when assessing the career stage and track record of a disabled researcher.

- While researchers can state they will be working part-time on a research grant, this must be justified. This requires the researcher to detail their specific disability and requirements around medical leave with the same consequences described in $\$ 2.2$, $\S 2.3$ and $\S 3.1$.

- Disabled doctoral candidates are not entitled to statutory sick pay following their PhD award due to the lack of national insurance contributions.

- Researchers funded by international fellowships sponsored by research councils do not have access to free healthcare if they have a pre-existing health condition. This leads to increased medical leave without sick pay.

\subsection{Track record}

The success rate of an individual researcher correlates substantially with their track record and career progression. Evidencing a strong track record forms a key part of the grant application process and is used, alongside the quality of the proposed research, to determine how successful a proposal will be if funded. Having a strong track record with numerous highimpact publications and research awards is seen to mitigate risk associated with the research proposal. In particular, individual fellowships are primarily judged on the candidate's leadership potential, which is evidenced by their track record. When assessing track record, panels and reviewers often do not take into account alternative career progressions (e.g. parttime work, medical leave, see §3.2). This can have the following effect on track record:

- Reduced number of publications in high-impact journals - Medical leave, part-time working and inaccessible lab equipment and facilities can drastically impact research output. While researchers produce high-quality research, it can often take longer due to the extra assistance required and the time taken off for medical leave. Furthermore, inaccessibility in journal submission (e.g. websites incompatible with screen-readers, inaccessible submission guidance) can lead to a lower publication rate.

- Lack of international recognition- Inability to access international conferences and to network effectively with international colleagues isolates disabled researchers. It prevents them from engaging with the international community and gaining both national and international recognition, which harms their track record.

- Reduced number of invited talks - Barriers to networking with the international community and attending conferences also leads to a lack of invited talks at key 
conferences in the respective research field. The majority of international conferences are hosted at inaccessible venues and do not have provision for remote access (via skype or teleconference). Increased travel costs (which are often not reimbursed by research councils) also prevent disabled researchers accessing conferences and thereby receiving invitations to present their work.

- Slow career progression and lack of promotion/recognition - As the above are measures of research success, disabled researchers are often passed over for promotion and do not gain significant recognition for their work. Compared to nondisabled researchers, they will often have fewer research outputs for their career stage due to the barriers described above (e.g. part-time work). This will lead to a slower career progression compared to non-disabled researchers, which impacts on the track record of the researcher.

\subsection{Impact of research frameworks:}

Research frameworks introduced by the government, such as REF/TEF, also serve as a measure of a researcher's success or track record. These frameworks are designed to measure and rank the research/teaching output of a university. For research, the success of a university is measured by the number of high-quality publications (ranked by impact factor of journal, number of citations and associated impact e.g. awards) and impact cases (e.g. commercialisation of research). These metrics are inequitable and inherently biased, rewarding a certain type of researcher (usually white, cis-male, non-disabled). They do not take into account alternative career paths, career breaks and part-time working due to disability/health issues and do not consider factors that have a detrimental impact on a researcher's ability to achieve these metrics (described in §3.3). These national frameworks therefore do not promote an inclusive research environment and perpetuate bias within the STEM environment.

\subsection{Institutional barriers:}

As demonstrated in $\S 3.1-\S 3.4$, disabled researchers experience several indirect barriers to obtain research funding within the research councils themselves, the STEM community and within national frameworks. However, they also experience indirect barriers within their own institutions and when visiting other research facilities that can impact on their ability to apply, obtain and utilise research funding. These barriers are as follows:

- Although it is stated in the Equality Act 2010 that employers have a legal duty to provide reasonable adjustments, disabled researchers often rely on the AtW or DSA scheme to fund equipment and support. These schemes have a long waiting time with researchers reporting waiting up to 1 year before receiving the equipment they need. They are also not designed to accommodate research and often do not provide funding for specialised research equipment (e.g. fume cupboard accessible to wheelchair users). Furthermore, they both place the substantial and ongoing burden of administration of evidencing needs, coordinating support and chasing multiple providers onto the $\mathrm{PhD}$ candidate or researcher, unremunerated. The reliance on these schemes negatively impacts livelihood, research time and progress, preventing disabled PhD students from completing their doctoral studies and causing disabled researchers leaving STEM completely.

- Research facilities and laboratories are inaccessible and do not have basic accessibility included at design stage. The majority of facilities do not meet the legal requirements detailed in British Standards 8300 ([8] BS EN 17210) and do not provide wheelchair access.

- Requests for building changes to existing research facilities to provide basic accessibility (in line with Equality Act 2010) are often rejected due to time constraints and cost. Even in the case of offering laboratory space to a new academic, changes to the existing infrastructure are rejected. Disabled researchers are instead offered research assistants to carry out the work on their behalf. 
- Standard lab equipment available is inaccessible. For example, for an individual who is blind in one eye, the use of microscopes becomes problematic. Specialist accessible lab equipment is either not available or extremely costly. The extra cost associated with this specialist equipment is not covered by the institution or the AtW/DSA schemes. Costs must be requested on grant applications, which requires the disabled researcher to justify the costs by detailing their disability and increases the overall cost of the grant.

- A large number of researchers conduct field trips as part of their research programmes. Equipment used for field trips is often inaccessible and specialist equipment either does not exist or is expensive. Costs for specialised equipment for field work are not covered by university or AtW and have to be requested on grant applications with the same caveats as described above.

- International conferences, national network meetings and events hosted by research councils are inaccessible. For example, venues often do not cater for wheelchair access, have telecoil loops and live subtitling, or have quiet spaces and rest rooms. This problem has already been raised by several groups. More information and guides to best practise can be found here in References [9] and [10].

- Disabled researchers often need remote access to meetings. Meeting rooms are not set up for teleconference/skype and detailed transcripts of meeting are not provided, excluding disabled researchers.

- The timing of lectures, appointments and meetings (10 minutes transition time) is not suitable for disabled researchers, who need extra time to travel between venues.

- The deadline for completing a PhD is now set to 4 years and has a clear cut-off period after this time. This drastically impacts disabled PhD students, who may take more time to complete a $\mathrm{PhD}$ (e.g. delay in receiving DSA, specialist equipment and reasonable adjustments, medical leave).

- Structural barriers can lead to a disabled researcher having a non-linear career trajectory, however there is a lack of flexibility in job experience to ensure these researchers stay in STEM employment.

\subsection{Unconscious bias:}

Unconscious bias is an association or assumption held by an individual that affects their attitudes and behaviours. It occurs when there is a stereotype associated with a certain characteristic that influences that individual's behaviour. Unconscious bias predominantly affects individuals that fall within underrepresented groups due to a protected characteristic (gender, race, age, sexual orientation and disability). While unconscious bias is often displayed in a subtle manner without intention, it can have a profound on the individual. When unconscious bias presents itself within the research funding process, it hinders a researcher's change of obtaining research funding and progressing in their career. The following examples of unconscious bias present significant barriers to disabled researchers:

- There is a lack of awareness of hidden disabilities, such as sensory impairments, which leads to issues of hostility and misunderstanding (e.g. 'just pretending'). These individuals face judgement on their use of resources and often do not receive the support they need.

- In particular, there is a lack of awareness of mental health issues (especially anxiety and depression) and neurodiversity (autism, Asperger's syndrome, dyslexia and dyspraxia) that can lead to hostility and judgement. Mental health issues and neurodiversity are also not considered disabilities by a majority of institutions, leading to a lack of support and understanding for these individuals. It is important to note that this report includes mental health issues and neurodiversity in our definition of disability. 
- Chronic illness (e.g. cancer, Crohn's disease) is again not considered a disability by a majority of institutions. As a result, support and extra assistance is not provided alongside medical leave.

- Stereotypes of disability are forged by the medical model, which sees the disability as a medical problem to try and solve. This directly leads to unconscious bias with individuals believing that having a disability prevents you from holding a job, performing sufficiently within a role and will never be able to outperform a non-disabled individual. One common misassumption is that all disabled individuals have learning disabilities, which affects their ability to perform in their job. In contrast, the social model states that people are disabled by barriers in society, not by their impairment or difference. Barriers can be physical (e.g. lack of accessible toilets) or caused by unconscious bias, (e.g. assumption of lower intelligence).

- Stereotypes of lower intelligence, slower output and lower efficiency for disabled researchers have a detrimental impact on research funding, as it is assumed that they cannot do 'excellent' research.

- For each new value/venue or new group of people, a disabled researcher has to ask for assistance. However, this is an embarrassing process that often leads to the disabled researcher gaining a label of 'attention-seeking', especially when a researcher possesses a hidden disability.

\subsection{Awareness and training:}

Having highlighted the issues surrounding unconscious bias in $\S 3.6$, it is important that awareness training is developed and implemented. In particular, the following should be addressed:

- There is a lack of representation of disabled researchers and training of panel members. Interview panel members are often not equipped to abide by/provide reasonable adjustments.

- Supervisors do not have adequate knowledge / experience with a disability to support disabled PhD students or postdoctoral researchers and no training is available to raise awareness of these issues.

- There is a lack of mentoring schemes specifically aimed for disabled researchers that can counter their lack of networking due to inaccessibility in conferences, etc. In particular, there is a lack of leadership training for disabled academics.

\section{Intersectionality}

It is important to consider intersectionality when addressing barriers within STEM for disabled researchers. An individual may be a member of more than one underrepresented group (e.g. BAME and disabled) and experience barriers associated with both protected characteristics. This will place the individual at a more severe disadvantage and amplify barriers associated with disability (e.g. chronic fatigue due to disability could be amplified for a woman on her period). Disabled researchers will also experience barriers and issues that are common to other underrepresented groups, including gender, BAME and low socioeconomic status ([1921]). Barriers and issues that bear intersectionality are identified below:

- Barriers at early stages in the research funding process affect carrier progression later on. For example, not obtaining research funding affects a researcher's ability to publish research, which in turn affects their track record and decreases their chance of success in obtaining research funding.

- Disabled researchers are primarily placed in supporting roles (e.g. Co-I) rather than encouraged to lead (e.g. PI).

- Universal metrics that are used to determine a researcher's success (e.g. number of publications, awards and honours) are not equitable across all groups, as some groups have barriers to productivity (e.g. career breaks due to maternity leave or ill health). 
- Socio-economic status and personal circumstances can play a significant role on an individual's ability to succeed. For example, a high socio-economic status can enable an individual to afford extra tuition (e.g. in British Sign Language) or purchase specialised research equipment, which can reduce the associated barrier.

- Some effects of disabilities are amplified by gender (e.g. chronic fatigue for women on period) that can hinder an individual's ability to progress without reasonable adjustments.

- The Equality Act 2010 applies not just for disabled researchers but any individual. It states that any discrimination due to a protected characteristic (sexual orientation, gender, age, race, disability) is illegal and reasonable adjustments should be made to ensure an inclusive environment for all.

\section{Suggested Actions}

Having identified the above barriers, we propose the following actions be taken to ensure the research funding process is accessible for disabled researchers. While this is not an exhaustive list of suggested actions, we believe that these actions will make an immediate difference to the careers and lives of disabled researchers.

1. Redesign research council portals and websites for accessibility, ensuring compatibility with screen-readers and easy wayfinding.

2. Provide all guidance in an accessible PDF format, with alternative text included for figures and transcripts or subtitles included for videos.

3. Advertise new grant schemes in a range of accessible formats.

4. Provide different means of communication (e.g. phone, email) for contacting research councils to ask questions about research funding schemes.

5. Make the required format and documentation consistent across all grant schemes and research councils.

6. Eliminate short turnaround times and deadlines (e.g. 1 week for a response to reviewers) within the grant process.

7. Allow applications across all research councils to be saved in instalments so as not to disadvantage people who need to pace computer use.

8. Ensure that disabled researchers are represented on interview panels and peer review panels.

9. Allow extra time for the interview process for disabled researchers and ensure that all reasonable adjustments required are provided.

10. Provide disability awareness and unconscious bias training to all members of interview and peer review panels.

11. Allow extra costs for reasonable adjustments outside of normal working routine on grants (e.g. travel costs for conferences, specialist research equipment, research assistant).

12. Maintain anonymity of disabled researcher in justification of resources and interview process by implementing two stage process to the application: Case for Support or proposed research blind-reviewed first before Justification of Resources is considered.

13. Consider alternative career paths (e.g. part-time) and career breaks (e.g. medical leave) when assessing the track record of researcher.

14. Enquiry into the efficacy of the research council DSA system in removing barriers for disabled doctoral candidates.

15. Implement a time limit on the implementation of reasonable adjustments to prevent these time costs being passed on to candidates. When there is a significant delay in implementation of reasonable adjustments (via DSA or AtW), provide an extension on the research grant or allow the grant to be postponed to account for the missed time.

16. Provide disability awareness training and extra support for principal investigators who take on a disabled PhD student.

17. Remove the practice of a writing-up year that comes without a living stipend. 
18. Resource reasonable adjustments for disabled candidates after the period of doctoral funding, during writing up, viva and corrections as policy within research councils.

19. Provide additional stipends for disabled grant holders where there are higher direct living costs arising from disability or reduced hours due to medical grounds that reduces earning capacity.

20. Pay national insurance contributions on stipends, so candidates are eligible for statutory sick pay following the award.

21. Provide access to healthcare for candidates with pre-existing health conditions on international fellowships when they cannot otherwise be insured.

22. Provide reasonable adjustments for potential candidates whilst applying and grant writing for other research council awards.

23. Modify fellowship structures that do not count as formal employment to provide improved rights under AtW.

24. Publish and provide open access to all current data relating to disability obtained by research councils to further identify barriers within the system and to monitor the impact of these suggested actions on the representation and success rate of disabled researchers.

25. 'Nothing about us without us': Form a focus/consultancy group of disabled researchers to ensure and monitor accessibility and inclusion within the research funding process.

\section{References}

1. https://www.hesa.ac.uk/data-and-analysis/staff

2. https://www.gov.uk/government/collections/family-resources-survey--2

3. Saxena, Procedia Economics and Finance, Volume 11, 2014, Pages 76-85

4. https://www.parliament.uk/documents/commons-committees/sciencetechnology/Correspondence/191030-

UKRI\%20to\%20Chair\%20re\%20Impact\%20of\%20funding\%20on\%20equality\%20div ersity\%20inclusion\%20and\%20accessibility.pdf

5. https://gds.blog.gov.uk/2018/07/16/why-gov-uk-content-should-be-published-in-htmland-not-pdf/

6. https://www.gov.uk/guidance/make-your-website-or-app-accessible-and-publish-anaccessibility-statement?utm_source=CampaignPage1\&utm_campaign=access_regs

7. Corlett, S. and Williams, J. (2011). "The effects of discourse and local organizing practices on disabled academics identities". CMS7 2011: The 7th International Critical Management Studies Conference, 11-13 July 2011, Naples, Italy. Available at http://nrl.northumbria.ac.uk/8651/

8. https://standardsdevelopment.bsigroup.com/committees/50001858

9. Crooks et al. (2011). "Accommodating Academics with Disabilities and Ongoing Health Concerns in the Workplace - A report on Canadian Universities". Available at https://www.academia.edu/778146/Accommodating_Academics_with_Disabilities_an d_Ongoing_Health_Concerns_in_the_Workplace_A_report_on_Canadian_Universiti es

10. Dali, K. (2018). "The right to be included: Ensuring the inclusive learning and work environment for people with disabilities in academia", Information and Learning Science. Volume 119, Number 9/10, Pages 486-513. Available at https://www.emerald.com/insight/content/doi/10.1108/lLS-04-2018-0032/full/html

11. Finkelstein, V. (1996). "Outside, 'Inside Out". Coalition GMCDP, Pages 30-36, https://disability-studies.leeds.ac.uk/wp-content/uploads/sites/40/library/finkelsteinInside-Out.pdf

12. French, S. (1998). "Surviving the Institution: working as a visually disabled lecturer in Higher Education". In Danusia Malina and Sian Maslin-Prothero (Eds),

13. Gibson, R. (1996). "Deaf Women Academics in Higher Education". 
14. Groth et al. (2017). "Participation of academics with disabilities in science using the example of AKTIF project." SHS Web of Conferences, Volume 37, Available at https://www.shs-

conferences.org/articles/shsconf/abs/2017/05/shsconf_erpa2017_01084/shsconf_erp a2017_01084.html

15. Horton, J. and Tucker, F. (2014) 'Disabilities in academic workplaces: experiences of human and physical geographers.' Transactions of The Institute of British Geographers. Volume 39, Issue, Pages 76-89. Available at https://rgsibg.onlinelibrary.wiley.com/doi/abs/10.1111/tran.12009

16. Kitchin, R. (2000). "The Researched Opinions on Research: Disabled people and disability research". Disability \& Society, Volume 15, Issue 1, Pages 25-47. Available at https://www.tandfonline.com/doi/abs/10.1080/09687590025757

17. Marvin, S. and Williams, J. (2015). "Impairment effects as a career boundary: a case study of disabled academics." Studies in Higher Education, Volume 40, No. 1, Pages 123-141, https://www.tandfonline.com/doi/abs/10.1080/03075079.2013.818637?journalCode= cshe20

18. Sang, K. (2017). "Disability and academic careers." Available at https://migrantacademics.files.wordpress.com/2017/05/disability-sang-may-2017.pdf

19. V. Walsh and L. Morley (1996): "Breaking Boundaries: Women in Higher Education" (Pages 177-183). London: Taylor \& Francis. https://files.eric.ed.gov/fulltext/ED41863

20. lantaffi, A. (1996). "Women and Disability in Higher Education: A literature search." In Val Walsh and Louise Morley (Eds.)

21. French, S. (1998): "Surviving the academy: feminist perspectives" (Pages 34-42). London: Taylor \& Francis. 